\title{
Risco climático para ocorrência de doenças fúngicas da videira no Sul e Sudeste do Brasil
}

\author{
Climatic risk for the occurrence of grapevine fungal diseases in South and Southeast Brazil
}

\author{
Iris Satie Hayashi Shimano² e Paulo Cesar Sentelhas ${ }^{3 *}$
}

\begin{abstract}
RESUMO - A ocorrência de doenças fúngicas na videira é fortemente influenciada pelas condições climáticas. No Brasil, a videira Vitis labrusca é cultivada em diversas regiões, desde o Rio Grande do Sul até o norte de Minas Gerais, as quais apresentam distintas características climáticas e, consequentemente, requerem diferentes estratégias de controle das doenças fúngicas. Com base nisto, o objetivo deste trabalho foi determinar o risco climático de ocorrência de doenças fúngicas em diferentes regiões produtoras do Sul e Sudeste do Brasil, utilizando um modelo de previsão de pulverizações, baseado na ocorrência de chuva. Para tanto, foram empregados dados pluviométricos diários de séries históricas de 30 anos das regiões de Manga (MG), Jales (SP), Jundiaí (SP), São Miguel Arcanjo (SP), Marialva (PR) e Caxias do Sul (RS). Foi determinado o número de pulverizações pelo sistema do calendário $\left(N P_{\text {calendário }}\right)$ e pelo sistema pluviométrico $\left(N P_{\text {chuva }}\right)$, em função da duração do ciclo em cada localidade para os diferentes períodos de safra, referentes a cada época de poda. Em Caxias do Sul, onde as chuvas são mais abundantes e bem distribuídas, o RC $\left[=\left(N P_{\text {chuva }} / N P_{\text {calendário }}\right) * 100\right]$ variou de 50 a $55 \%$ entre as podas de agosto e setembro, enquanto que na região de Manga o RC variou de $1 \%$ nas podas de maio a $24 \%$ para as podas de fevereiro. Nas demais regiões, o RC apresentou valores entre 16 e 56\%, demonstrando que o risco de ocorrência dessas doenças é variável com o clima e que o monitoramento das condições meteorológicas pode auxiliar para a recomendação racional de pulverizações para o controle de doenças fúngicas na videira Vitis labrusca.
\end{abstract}

Palavras-chave: Videira. Plantas-doenças e pragas. Vegetação e clima.

\begin{abstract}
The occurrence of fungal diseases in the grapevine is strongly influenced by climatic conditions. In Brazil, the grapevine Vitis labrusca, is grown in several regions, from Rio Grande do Sul to the north of Minas Gerais, which present distinct climatic conditions therefore requiring different strategies for the control of fungal diseases. Based on that, the aim of this study was to determine climatic risk in the occurrence of fungal diseases in different productive regions of the South and Southeast of Brazil, using a prediction model for spraying, based on rainfall occurrence. In order to do this, daily rainfall data from the regions of Manga (MG), Jales (SP), Jundiaí (SP), São Miguel Arcanjo (SP), Marialva (PR) and Caxias do Sul (RS), taken from a thirty-year time series were used. The number of sprays was determined using the calendar $\left(N S_{\text {calendar }}\right)$ and rainfall $\left(N S_{\text {rainfall }}\right)$ sytems, as a function of the duration of every harvest cycle for each location, as related to the pruning periods. In Caxias do Sul, where rainfall is more abundant and better distributed, the RC [ $=\left(N S_{\text {rainfall }} / N S_{\text {calendar }} * 100\right]$, varied from 50 to 55\% for pruning dates from August to September, whereas in the Manga region, the $\mathrm{RC}$ ranged from $1 \%$ for pruning carried out in May to 24\% for pruning in February. In the remaining regions, the RC values were between 16 and $56 \%$, demonstrating that the risk of occurrence of these diseases varies with the weather, and that the monitoring of weather conditions can help in a rational recommendation of spraying frequency in the control of fungal diseases in the grapevine Vitis labrusca.
\end{abstract}

Key words: Grapevine. Plant diseases and pests. Vegetation and climate.

\footnotetext{
*Autor para correspondência

${ }^{1}$ Recebido para publicação em 27/03/2012; aprovado em 26/12/2012

Baseado no Trabalho de Conclusão de curso da primeira autora, para a obtenção do título de Engenheiro Agrônomo, junto à ESALQ/USP ${ }^{2}$ Estagiária do Departamento de Engenharia de Biossistemas, ESALQ/USP, Caixa Postal 11, Piracicaba-SP, Brasil, 13.418-900, irishimano@ yahoo.com.br. ${ }^{3}$ Bolsista de Produtividade do CNPq, Departamento de Engenharia de Biossistemas, ESALQ/USP, Caixa Postal 11, Piracicaba-SP, Brasil, 13.418-900, pcsentel.esalq@usp.br
} 


\section{INTRODUÇÃO}

A videira é produzida em diversas condições climáticas no Brasil, destacando-se nos estados de São Paulo, Minas Gerais, Paraná, Rio Grande do Sul, Pernambuco e Bahia. Nessas áreas, as doenças fúngicas correspondem a um dos principais problemas enfrentados pelos viticultores. $\mathrm{O}$ controle dessas doenças, no entanto, é realizado de maneira similar em todas as regiões, com aplicações semanais de fungicidas, o que eleva os custos de produção, assim como os riscos de contaminação ambiental e humana.

A ocorrência de doenças em plantas é resultado de uma complexa interação entre patógeno, hospedeiro e ambiente (VALE; JESUS JÚNIOR; ZAMBOLIM, 2004). Baseado nesse princípio, vários sistemas de alerta fitossanitário vêm sendo desenvolvidos, os quais visam à racionalização do uso de defensivos agrícolas, com base nas condições meteorológicas vigentes ao longo do ciclo (PEREIRA; ANGELOCCI; SENTELHAS, 2002).

Diversos sistemas de alerta fitossanitário existem para auxiliar no controle de doenças fúngicas da cultura da videira. Na sua grande maioria, consideram aspectos relativos aos processos biológicos dos fungos e as influências do ambiente nesses processos, como os modelos desenvolvidos para simular o desenvolvimento do míldio da videira Vitis vinifera (CAFFI et al., 2007; ORLANDINI; MASSETTI; DALLA MARTA, 2008; ROSSI et al., 2005). Devido às suas complexidades, muitas vezes esses modelos têm sua aplicação limitada pela ausência de dados de entrada, especialmente dados meteorológicos como a duração do período de molhamento. Nesse sentido, modelos menos complexos, que consideram variáveis ambientais facilmente disponíveis (como a chuva), possuem uma grande vantagem, já que podem ser aplicados em larga escala.

Um exemplo de um modelo simples foi desenvolvido por Pedro Júnior et al. (1999). Esses autores avaliaram o uso da chuva para a previsão de épocas de pulverizações para o controle do míldio, da antracnose e da mancha-das-folhas em videira "Niagara Rosada" e obtiveram como resultado um controle satisfatório com aplicações de fungicidas após a ocorrência de $20 \mathrm{~mm}$ de chuva. Esse sistema proporcionou reduções médias da ordem de $40 \%$ no número total de pulverizações quando comparado ao sistema convencional, sem haver redução significativa da produtividade e qualidade das uvas.

O sistema pluviométrico de recomendação de pulverizações de Pedro Júnior, Pezzopane e Martins (1999) foi empregado por Bardin, Pedro Júnior e Moraes (2010) para a caracterização espacial do risco climático de ocorrência de doenças fúngicas da videira "Niagara Rosada", em diferentes épocas de poda, na região de Jundiaí, no Estado de São Paulo. Esse estudo demonstrou que o uso do modelo permitiu verificar as variações espaciais do risco de doenças, tomando por base a relação entre o número de pulverizações recomendado pelo sistema pluviométrico e o número de pulverizações estabelecido pelo calendário.

Sendo assim, o conhecimento das condições climáticas adequadas ao estabelecimento e desenvolvimento dos patógenos permite inferir sobre o risco de ocorrência de doenças em uma determinada época e localidade. $\mathrm{O}$ uso de séries históricas de dados meteorológicos aplicados a modelos de previsão de doenças pode, então, ser útil para se caracterizar a variabilidade espacial e interanual da ocorrência de doenças, possibilitando um melhor planejamento das estratégias de manejo das fitopatologias.

Dentro desse contexto, o objetivo deste trabalho foi avaliar o risco climático de ocorrência de doenças fúngicas na videira Vitis labrusca para os principais períodos de safra das diferentes regiões produtoras do Sul e do Sudeste do Brasil.

\section{MATERIAL E MÉTODOS}

As localidades empregadas no presente estudo foram: Caxias do Sul, RS; Marialva, PR; Manga, MG; Jundiaí, São Miguel Arcanjo e Jales, SP (Tabela 1). Foram utilizados dados diários de precipitação pluvial (mm) disponíveis no Sistema de Informações Hidrológicas da Agência Nacional de Águas (HIDROWEB). As séries históricas utilizadas possuíam 30 anos de dados de chuva para cada uma das localidades, como apresentado na Tabela 1.

As temperaturas médias e as precipitações pluviométricas médias anuais de cada localidade são apresentadas na Tabela 2, na qual pode se observar as diferenças entre as regiões analisadas, com o clima variando, de acordo com a classificação climática de Köppen (PEREIRA; ANGELOCCI; SENTELHAS , 2002), de sub-tropical úmido (Cfa), em Caxias do Sul, RS, a tropical sub-úmido (Aw), em Manga, MG.

Quando alguma estação apresentava falhas na série de dados, os dados faltantes foram preenchidos com dados oriundos da estação mais próxima, empregando-se os procedimentos recomendados por Lozada Garcia et al. (2006).

A duração do ciclo poda-colheita foi estimada, para cada localidade e época de poda, pelo somatório de graus dia (GD). Para tanto, estimou-se as temperaturas médias mensais do ar para cada localidade por meio das equações propostas por Alvares et al. (2011), em que se consideram apenas as coordenadas geográficas e a altitude. A necessidade térmica considerada para a videira Vitis labrusca, cultivar "Niagara Rosada", foi de $1.550{ }^{\circ} \mathrm{C}$ $\mathrm{dia}^{-1}$ para uma temperatura-base inferior igual a $10{ }^{\circ} \mathrm{C}$ (PEDRO JÚNIOR; SENTELHAS; POMMER, 1994). 
Tabela 1 - Coordenadas geográficas, altitude e séries históricas de dados pluviométricos diários das localidades utilizadas no presente estudo

\begin{tabular}{lcccc}
\hline Local/Estado & Latitude $(\mathrm{S})$ & Longitude $(\mathrm{W})$ & Altitude $(\mathrm{m})$ & Série histórica* $^{*}$ \\
\hline Manga, MG & $14^{\circ} 45^{\prime}$ & $43^{\circ} 56^{\prime}$ & 454 & $1981-2010$ \\
Jales, SP & $20^{\circ} 26^{\prime}$ & $50^{\circ} 32^{\prime}$ & 380 & $1975-2004$ \\
Jundiaí, SP & $23^{\circ} 11^{\prime}$ & $46^{\circ} 53^{\prime}$ & 700 & $1981-2010$ \\
São Miguel Arcanjo, SP & $23^{\circ} 52^{\prime}$ & $48^{\circ} 00^{\prime}$ & 667 & $1975-2004$ \\
Marialva, PR & $23^{\circ} 36^{\prime}$ & $51^{\circ} 51^{\prime}$ & 448 & $1981-2010$ \\
Caxias do Sul, RS & $29^{\circ} 11^{\prime}$ & $51^{\circ} 11^{\prime}$ & 730 & $1981-2010$ \\
\hline
\end{tabular}

*Fonte: http://hidroweb.ana.gov.br

Tabela 2 - Valores normais da temperatura média anual (Tmed) e da precipitação pluviométrica anual (Prec) para as localidades utilizadas no presente estudo e seus respectivos climas de acordo com a classificação de Köppen

\begin{tabular}{lccc}
\hline \multicolumn{1}{c}{ Local/Estado } & Tmed $(\mathrm{oC})^{*}$ & Prec $(\mathrm{mm})^{* *}$ & Clima - Köppen*** \\
\hline Manga, MG & 24,2 & 783 & Aw - Tropical Sub-úmido \\
Jales, SP & 24,1 & 1317 & Aw - Tropical Úmido \\
Jundiaí, SP & 20,0 & 1487 & Cwa - Tropical de Altitude, Úmido \\
São Miguel Arcanjo, SP & 19,8 & 1332 & Cfa - Sub-tropical Úmido \\
Marialva, PR & 21,2 & 1644 & Cwa - Tropical de Altitude, Úmido \\
Caxias do Sul, RS & 15,5 & 1713 & Cfa - Sub-tropical Úmido \\
\hline
\end{tabular}

Fonte: *Alvares et al. (2012), **HIDROWEB/ANA e ***Pereira, Angelocci e Sentelhas (2002)

Foi feita uma média da duração do ciclo para cada localidade, obtendo-se um único valor que foi adotado para o cálculo do risco climático. Os valores adotados foram: 118 dias em Manga; 123 dias em Jales; 151 dias em Jundiaí; 155 dias para São Miguel Arcanjo; 129 dias em Marialva. Somente para o município de Caxias do Sul, no estado do Rio Grande do Sul, o ciclo não foi estimado pelo acúmulo de GD, já que este processo resultava em um valor muito longo e, portanto, irreal para a região. Nesse caso, adotou-se um valor médio de 153 dias, de acordo com o que é normalmente observado na região (SCHIEDECK et al., 1997).

As datas de poda adotadas para cada localidade (Tabela 3) foram determinadas de acordo com as práticas normalmente realizadas nessas regiões, de acordo com o apresentado em Pommer (2003).

O risco climático de ocorrência de doenças fúngicas na videira, que inclui o míldio (Plasmopara viticola (Berk. \& Curt.) Berl. of de Toni), a antracnose (Sphaceloma ampelinum de Bary) e a mancha-dasfolhas (Pseudocercospora vitis (Lév.) Speg.), foi calculado para cada uma das localidades em diferentes períodos de safra, referentes às épocas de poda, de acordo com a Equação 1, proposta por Lozada Garcia et al. (2008) e Bardin, Pedro Júnior e Moraes (2010):
$R C=\frac{N P_{\text {chuva }}}{N P_{\text {calendário }}} \times 100$

em que: $R C$ é o risco climático de ocorrência de doenças, expresso em porcentagem; $N P_{\text {chuva }}$ é o número de pulverizações necessárias de acordo com sistema pluviométrico (PEDRO JÚNIOR et al., 1999); e $N P_{\text {calendário }}$ é o número máximo de pulverizações possíveis baseadas no calendário, em função da duração do ciclo (poda-colheita).

Para a estimativa do número de pulverizações necessárias devido à ocorrência de chuva $\left(N P_{\text {chuva }}\right)$ foi utilizado o modelo pluviométrico desenvolvido por Pedro Júnior, Pezzopane e Martins (1999), no qual as aplicações de defensivos são recomendadas após a ocorrência de períodos que totalizem pelo menos $20 \mathrm{~mm}$ de chuva, respeitando a carência do produto de sete dias. Para tanto, foram empregados os dados diários de chuva das séries históricas de 30 anos para cada uma das localidades (Tabela1). O acúmulo de chuva para indicação de pulverização se iniciou 10 dias após a data de poda, sendo finalizada em 10 dias antes da data estimada de colheita.

O número de pulverizações necessárias pelo sistema do calendário $\left(N P_{\text {calendário }}\right)$ foi determinado para o período entre a poda e a colheita, considerando-se um intervalo de sete dias entre as pulverizações. 
Tabela 3 - Datas de poda (P) e de colheita (C) da videira consideradas para cada localidade para as simulações de ciclo e do risco climático de ocorrência de doenças

\begin{tabular}{|c|c|}
\hline Local/Estado & Datas \\
\hline \multirow{2}{*}{ Manga, MG } & P: $01 / 02,15 / 02,01 / 03,15 / 03,01 / 04,15 / 04,01 / 05,15 / 05$ \\
\hline & C: $30 / 05,13 / 06,27 / 06,11 / 07,28 / 07,11 / 08,27 / 08,10 / 09$ \\
\hline \multirow{2}{*}{ Jales, SP } & P: 01/02, 15/02, 01/03, 15/03, 01/04, 15/04, 01/05, 15/05, 01/06, 15/06 \\
\hline & C: $04 / 06,18 / 06,02 / 07,16 / 07,02 / 08,16 / 08,01 / 09,15 / 09,02 / 10,16 / 10$ \\
\hline \multirow{2}{*}{ Jundiaí, SP } & P: 01/07, 15/07, 01/08, 15/08, 01/09, 15/09 \\
\hline & C: $29 / 11,13 / 12,30 / 12,13 / 01,30 / 01,13 / 01$ \\
\hline \multirow{2}{*}{ S. Miguel Arcanjo, SP } & P: 01/07, 15/07, 01/08, 15/08, 01/09, 15/09 \\
\hline & C: 03/12, 16/12, 01/01, 17/01, 03/02, 17/02 \\
\hline \multirow{2}{*}{ Marialva, PR } & P: 01/07, 15/07, 01/08, 15/08, 01/09, 15/09 \\
\hline & C: 07/11, 21/11, 08/12, 22/12,08/01, 22/01 \\
\hline \multirow{2}{*}{ Caxias do Sul, RS } & P: $01 / 08,15 / 08,01 / 09,15 / 09$ \\
\hline & C: $01 / 01,15 / 01,01 / 02,15 / 02$ \\
\hline
\end{tabular}

Para verificar o efeito da época de poda sobre o risco climático $(\mathrm{RC})$, foram calculadas a média e o desvio padrão do número de pulverizações $\left(N P_{\text {chuva }}\right)$ para cada localidade por época de poda, para os 30 anos das séries históricas. Para se avaliar a variabilidade interanual do RC foi calculada uma média entre as diferentes épocas de poda para cada localidade para cada ano da série histórica. Além disso, foram determinados os percentis de $50,70,80,90$ e $95 \%$ de probabilidade do RC para cada local.

Para a análise dos dados anuais e médios de RC, essa variável foi classificada em quatro classes, de acordo com a proposição dos seguintes intervalos: < $20 \%$ (Baixo); entre 21 e $40 \%$ (Moderado); entre 41 e $60 \%$ (Alto); e > 60\% (Muito Alto).

\section{RESULTADOS E DISCUSSÃO}

Os valores médios de número de pulverizações com base no sistema pluviométrico $\left(N P_{\text {chuva }}\right)$, desvio padrão, número de pulverizações baseadas no calendário $\left(N P_{\text {calendário }}\right)$ e risco climático médio de ocorrência de doenças (RC) para cada localidade e época de poda são apresentados nas Tabelas 4 e 5.

O número de pulverizações necessárias baseadas no calendário $\left(N P_{\text {calendário }}\right)$ foi calculado em função da duração dociclo, o qualé diretamente influenciado pela temperatura. Desse modo, locais com temperaturas mais elevadas resultaram em um ciclo menor e, consequentemente, em um $N P_{\text {calendário }}$ menor em comparação às regiões de temperaturas mais amenas (Tabela 4 e 5).
Pode-se observar que na região de Manga o $N P_{\text {chuva }}$ foi menor do que nas demais localidades, sendo que em alguns anos praticamente não foi recomendado nenhuma pulverização. Isso se deve ao fato de que nessa região as chuvas são menos freqüentes, já que a principal época de produção coincide com o inverno, ou seja, o período menos chuvoso do ano (Figura 1).

Em Jundiaí, São Miguel Arcanjo, Marialva e Caxias do Sul, as podas são realizadas no final do inverno, de modo que o ciclo de produção da videira se concentra na primavera/verão. Nesse período, as chuvas são mais abundantes e as temperaturas mais elevadas, o que torna o ambiente mais propício à ocorrência de doenças fúngicas, resultando em número de pulverizações mais elevado. Comparando as épocas de poda nessas regiões, observa-se que o número de pulverizações aumenta quanto mais tardia for a poda. Resultados semelhantes foram observados por Bardin, Pedro Júnior e Moraes (2010) no Pólo do circuito das frutas em São Paulo. Em Manga e Jales, no entanto, essa tendência não é observada, ocorrendo o contrário, pois no período de poda de fevereiro a junho, há uma diminuição das chuvas, sendo fevereiro e março as épocas mais chuvosas (Figura 1).

Em Marialva, o $N P_{\text {chuva }}$ foi menor em comparação ao de Jundiaí e de São Miguel Arcanjo para as mesmas épocas de poda. No entanto, o RC foi mais alto, o que se deveu basicamente ao menor $N P_{\text {calendário }}$ em Marialva, decorrente do ciclo mais curto da cultura nessa região.

Na Figura 2 é apresentada a variação interanual do risco climático de ocorrência de doenças fúngicas na videira em cada uma das localidades. Observa-se que há 
Tabela 4 - Valores médios de número de pulverizações devido à ocorrência de chuva $\left(N P_{\text {chuva }}\right)$, desvio padrão, número de pulverizações baseadas no calendário $\left(N P_{\text {calendário }}\right)$ e risco climático médio de ocorrência de doenças $(\mathrm{RC})$ na videira Vitis labrusca, nas localidades de Manga (MG), Jales e Jundiaí (SP)

\begin{tabular}{|c|c|c|c|c|c|}
\hline Local/Estado & Poda & NPchuva & Desvio Padrão & NPcalendário & $\mathrm{RC}(\%)$ \\
\hline \multirow{8}{*}{ Manga, MG } & $01 / \mathrm{Fev}$ & 3,33 & 1,21 & 14 & 23,81 \\
\hline & $15 / \mathrm{Fev}$ & 2,63 & 1,30 & 14 & 18,81 \\
\hline & 01/Mar & 2,07 & 1,17 & 14 & 14,76 \\
\hline & 15/Mar & 1,30 & 0,88 & 14 & 9,29 \\
\hline & $01 / \mathrm{Abr}$ & 0,57 & 0,68 & 14 & 4,05 \\
\hline & $15 / \mathrm{Abr}$ & 0,23 & 0,50 & 14 & 1,67 \\
\hline & 01/Mai & 0,17 & 0,38 & 14 & 1,19 \\
\hline & 15/Mai & 0,13 & 0,35 & 14 & 0,95 \\
\hline \multirow{10}{*}{ Jales, SP } & $01 / \mathrm{Fev}$ & 6,53 & 1,14 & 15 & 43,56 \\
\hline & $15 / \mathrm{Fev}$ & 5,83 & 1,23 & 15 & 38,89 \\
\hline & 01/Mar & 4,90 & 1,09 & 15 & 32,67 \\
\hline & 15/Mar & 4,07 & 1,01 & 15 & 27,11 \\
\hline & $01 / \mathrm{Abr}$ & 3,43 & 1,01 & 15 & 22,89 \\
\hline & $15 / \mathrm{Abr}$ & 2,70 & 1,02 & 15 & 18,00 \\
\hline & 01/Mai & 2,53 & 1,28 & 15 & 16,89 \\
\hline & 15/Mai & 2,53 & 1,36 & 15 & 16,89 \\
\hline & 01/Jun & 2,73 & 1,31 & 15 & 18,22 \\
\hline & $15 /$ Jun & 3,17 & 1,53 & 15 & 21,11 \\
\hline \multirow{6}{*}{ Jundiaí, SP } & 01/Jul & 6,53 & 1,55 & 19 & 34,39 \\
\hline & $15 / \mathrm{Jul}$ & 7,23 & 1,74 & 19 & 38,07 \\
\hline & 01/Ago & 8,30 & 1,53 & 19 & 43,68 \\
\hline & 15/Ago & 9,30 & 1,44 & 19 & 48,95 \\
\hline & $01 /$ Set & 10,30 & 1,58 & 19 & 54,21 \\
\hline & $15 /$ Set & 10,73 & 1,70 & 19 & 56,49 \\
\hline
\end{tabular}

Tabela 5 - Valores médios de número de pulverizações devido à ocorrência de chuva $\left(N P_{\text {chuva }}\right)$, desvio padrão, número de pulverizações baseadas no calendário $\left(N P_{\text {calendário }}\right.$ e risco climático médio de ocorrência de doenças (RC) na videira Vitis labrusca, nas localidades de São Miguel Arcanjo (SP), Marialva (PR) e Caxias do Sul (RS)

\begin{tabular}{|c|c|c|c|c|c|}
\hline Local/Estado & Poda & NPchuva & Desvio Padrão & NPcalendário & $\mathrm{RC}(\%)$ \\
\hline \multirow{6}{*}{ São Miguel Arcanjo, SP } & 01/Jul & 7,53 & 1,74 & 20 & 37,67 \\
\hline & $15 / \mathrm{Jul}$ & 8,23 & 1,81 & 20 & 41,17 \\
\hline & 01/Ago & 8,90 & 1,67 & 20 & 44,50 \\
\hline & 15/Ago & 9,70 & 1,68 & 20 & 48,50 \\
\hline & $01 /$ Set & 10,30 & 1,53 & 20 & 51,50 \\
\hline & $15 /$ Set & 10,73 & 1,44 & 20 & 53,67 \\
\hline \multirow{6}{*}{ Marialva, PR } & 01/Jul & 6,33 & 1,65 & 16 & 39,58 \\
\hline & $15 / \mathrm{Jul}$ & 6,87 & 1,74 & 16 & 42,92 \\
\hline & 01/Ago & 7,53 & 1,78 & 16 & 47,08 \\
\hline & 15/Ago & 8,10 & 1,56 & 16 & 50,63 \\
\hline & 01/Set & 8,90 & 1,27 & 16 & 55,63 \\
\hline & 15/Set & 9,00 & 1,26 & 16 & 56,25 \\
\hline \multirow{4}{*}{ Caxias do Sul, RS } & 01/Ago & 9,60 & 1,63 & 19 & 50,53 \\
\hline & 15/Ago & 9,77 & 1,72 & 19 & 51,40 \\
\hline & 01/Set & 10,40 & 1,85 & 19 & 54,74 \\
\hline & $15 /$ Set & 10,23 & 1,74 & 19 & 53,86 \\
\hline
\end{tabular}


uma relação direta, como era de se esperar, entre o RC e total anual pluviométrico (Figura 3). De modo geral, as regiões mais chuvosas apresentam os maiores valores de RC. Entretanto em Jales, apesar da precipitação total anual aproximar-se da observada em São Miguel Arcanjo, o RC foi consideravelmente menor, de modo que a época de poda e a distribuição da chuva ao longo do ano foram os fatores que mais influenciaram na quantidade de pulverizações requeridas, de acordo com o sistema pluviométrico.
De acordo com a Figura 2, ainda pode-se observarque há um aumento gradativo do RC de Manga para Caxias do Sul, com um aumento expressivo também na variabilidade interanual. Enquanto que em Manga o RC permanece sempre classificado como muito baixo, em Caxias do Sul, o RC oscila entre as classes moderado a muito alto. Como mencionado anteriormente, essas oscilações obedecem ao regime de chuvas, como mostra a Figura 3, assim como à época em que o ciclo da videira é conduzido.

Figura 1 - Precipitação mensal média para o período de 30 anos das séries históricas das localidades de Manga (MG), Jales (SP), Jundiaí (SP), São Miguel Arcanjo (SP), Marialva (PR) e Caxias do Sul (RS)
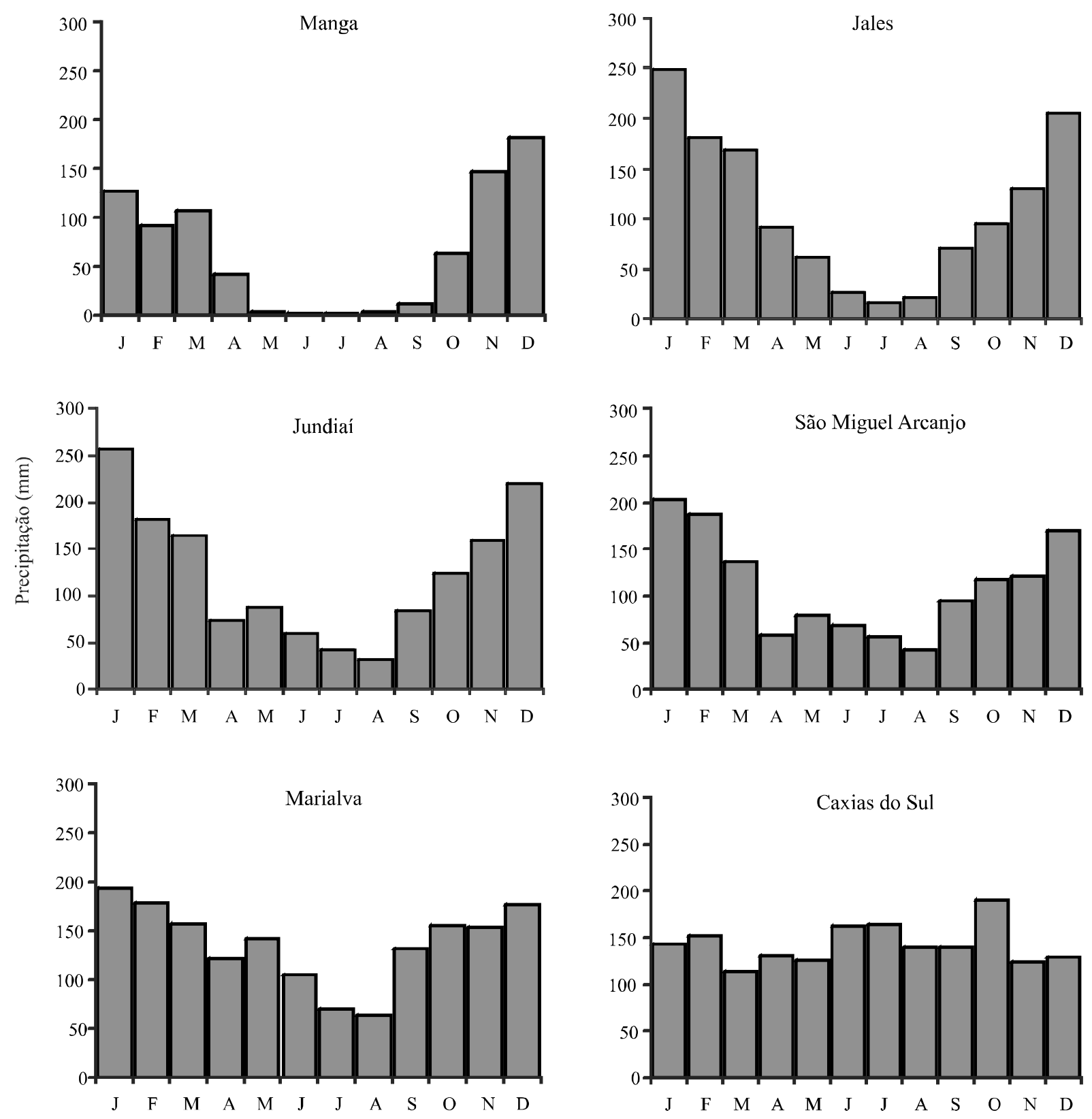
Figura 2 - Variação interanual do risco climático médio para a ocorrência de doenças fúngicas na videira Vitis labrusca nas localidades de Manga (MG), Jales (SP), Jundiaí (SP), São Miguel Arcanjo (SP), Marialva (PR) e Caxias do Sul (RS)
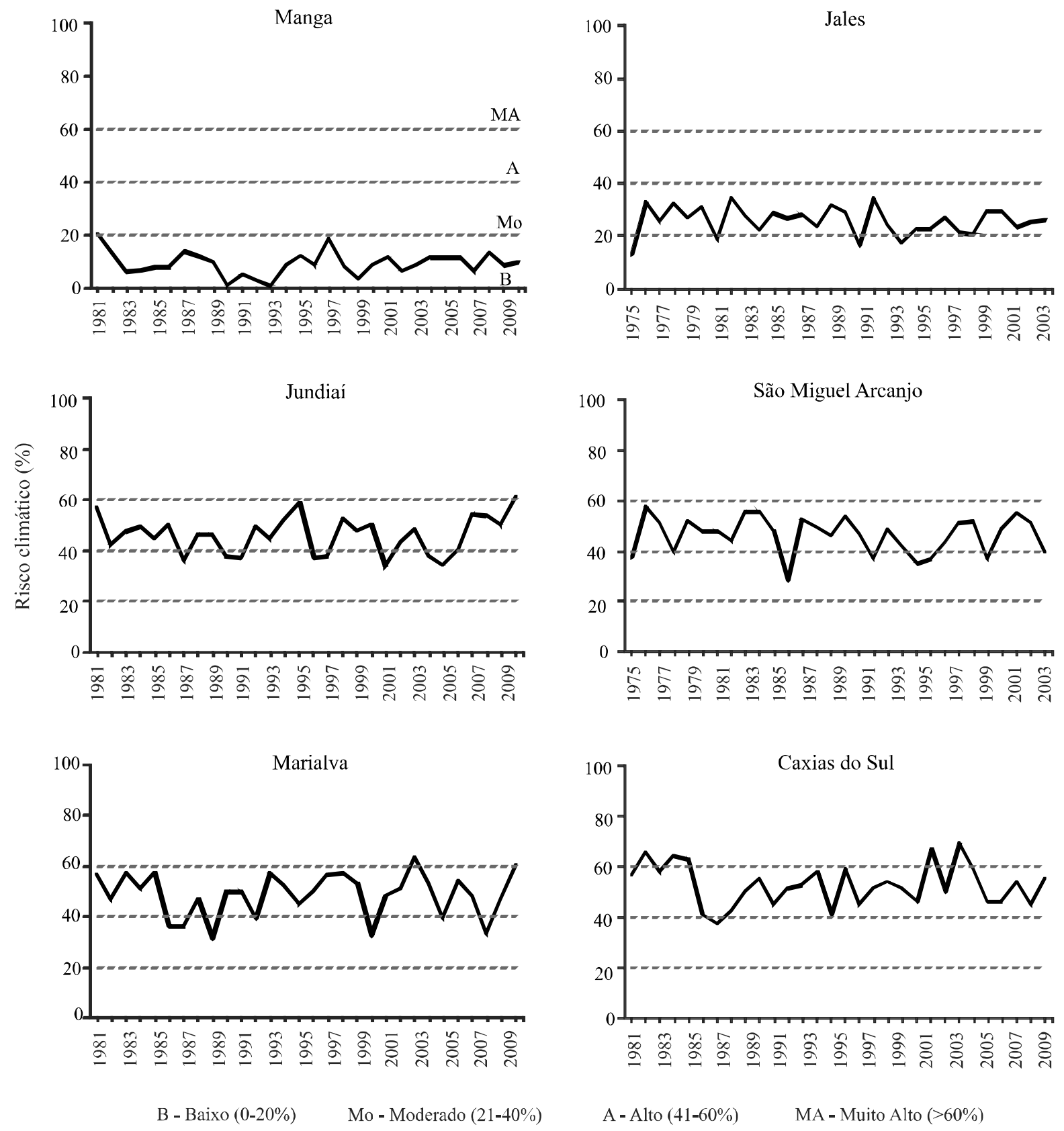

A - Alto (41-60\%) MA - Muito Alto $(>60 \%)$

Análise semelhante foi empregada por Lozada Garcia et al. (2008) para classificar diferentes ambientes de produção de batata quanto ao risco de ocorrência de Phytophtora infestans na região andina da Venezuela. Os autores observaram padrão de variabilidade do risco climático para a doença semelhante ao obtido no presente estudo, considerando-se diferentes épocas de semeadura. Com base nesse estudo, foram recomendadas as melhores épocas de semeadura para a batata nessa região, de modo a diminuir o número de pulverizações e assim reduzir o custo de produção.

Nas Tabelas 6 e 7 se encontram os valores dos percentis do RC para os níveis de 50; 70; 80; 90 e 95\% de probabilidade, em cada uma das localidades conforme as datas de poda simuladas. 
Figura 3 - Variação interanual do total anual de chuva nas localidades de Manga (MG), Jales (SP), Jundiaí (SP), São Miguel Arcanjo (SP), Marialva (PR) e Caxias do Sul (RS)
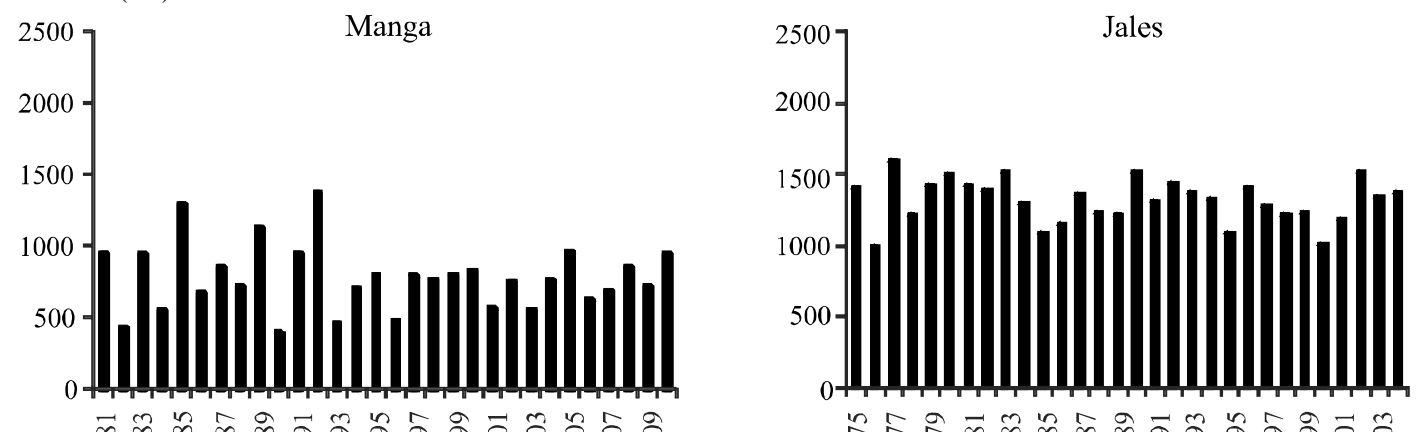

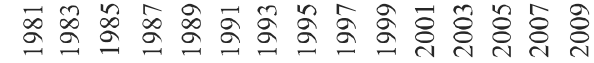

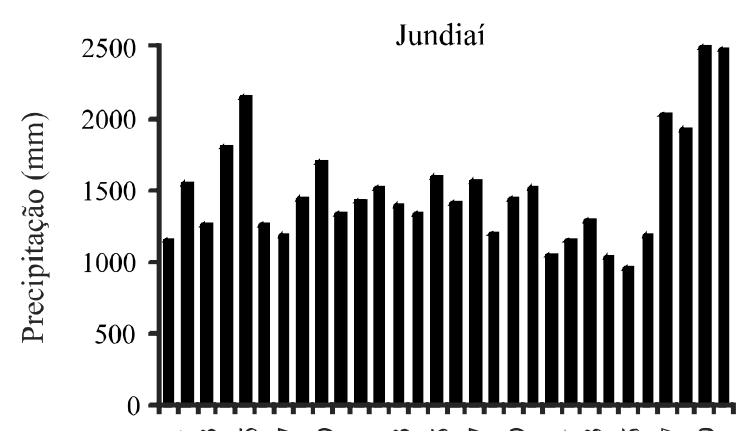

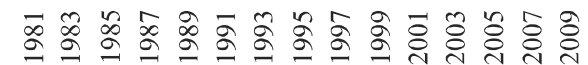
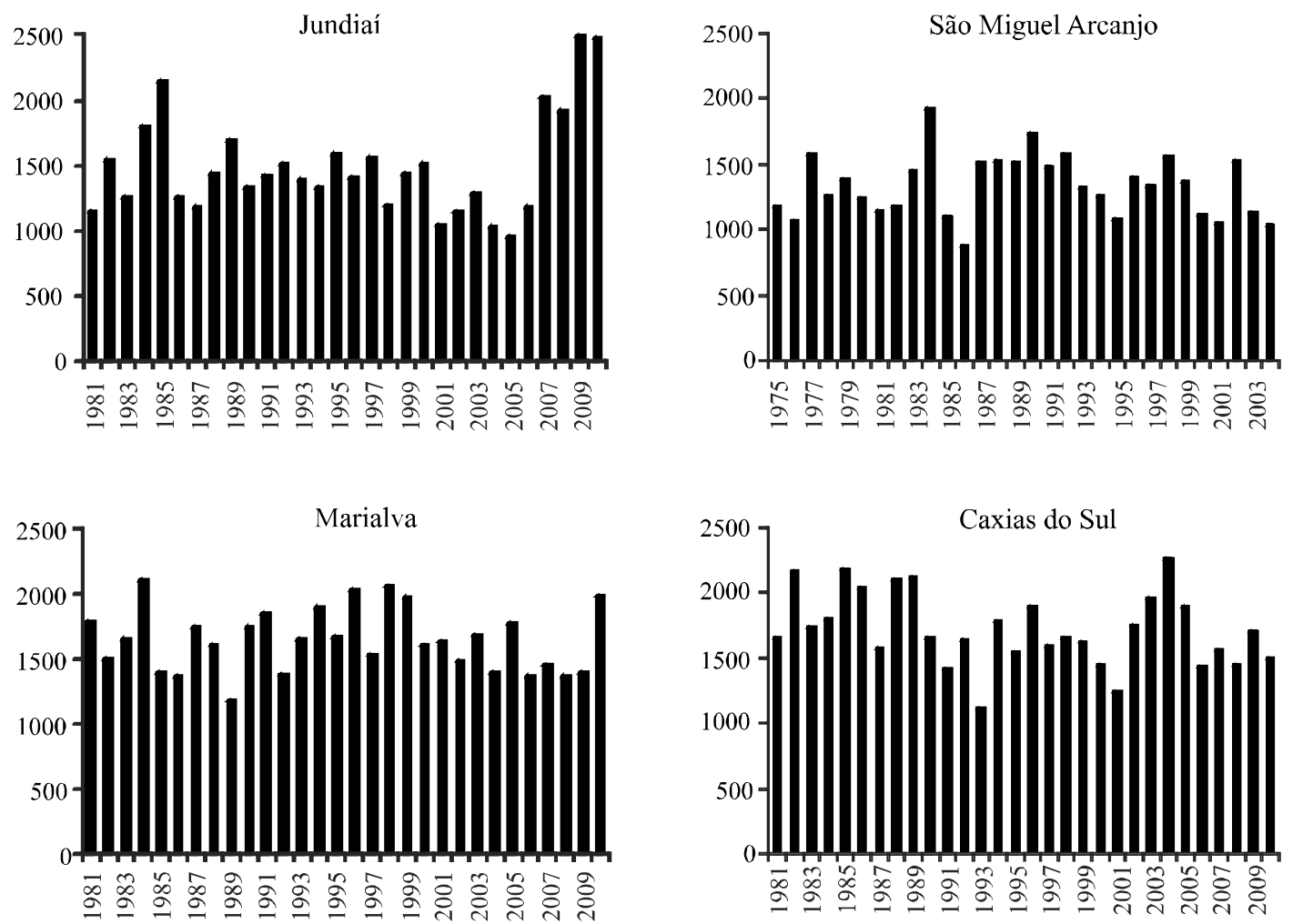

Em Manga (Tabela 6), para os períodos de poda realizados a partir de $1^{\circ}$ de abril, o RC foi considerado baixo com $90 \%$ de probabilidade de ocorrência de riscos menores que $14,29 \%$. Para as podas a partir de 15 de abril, observa-se que em $80 \%$ dos anos não há necessidade de pulverização para controle das doenças fúngicas já que as chuvas diminuem sensivelmente (Figura 1). Nas demais épocas de poda, o RC, em 95\% dos anos, situa-se entre baixo e moderado.

Jales é considerada uma região predominantemente de RC moderado, com $95 \%$ de probabilidade de ocorrência de riscos menores que $40 \%$ para podas realizadas a partir de março (Tabela 6). Para as demais épocas de poda, devido à maior ocorrência de chuvas, o RC passa a ser considerado

alto em $30 \%$ dos anos na poda de $1^{\circ}$ de fevereiro e em $20 \%$ dos anos na poda de 15 de fevereiro.

Na região de Jundiaí (Tabela 6), o RC varia de moderado à muito alto, havendo uma tendência de aumento do RC com o atraso da poda, já que as podas tardias fazem com que grande parte do ciclo ocorra durante a estação chuvosa (Figura 1). Observa-se na Tabela 5 que nas podas de $1^{\circ}$ e 15 de julho $90 \%$ dos anos tem RC alto, ao passo que nas podas tardias isso ocorre apenas em $70 \%$ dos anos na poda de $1^{\circ}$ de setembro e em $50 \%$ dos anos na poda de 15 de setembro. Essa situação é muito semelhante à que ocorre em São Miguel Arcanjo (Tabela 6), onde os valores de RC indicam que na grande maioria dos anos esse risco é alto. 
Em Marialva (Tabela 7), os valores de RC são um pouco mais acentuados do que nas regiões de Jundiaí e São Miguel Arcanjo, tendendo a se assemelharem mais às condições de Caxias do Sul, já que os regimes de chuva nessas regiões são similares (Figura 1).

Em Marialva, a partir da poda de $1^{\circ}$ de agosto começa a haver probabilidade de ocorrência de RC muito alto (5\%). Essa probabilidade aumenta $10 \%$ na poda de 15 de agosto e para $30 \%$ nas podas de setembro (Tabela 7). Situação semelhante é observada em Caixas do Sul, em que em $10 \%$ dos anos há chances de RC muito alto na poda de $1^{\circ}$ de agosto. Para as demais podas, a probabilidade de $\mathrm{RC}$ ser maior do que $60 \%$ sobe para $20 \%$.

Os dados de RC apresentados neste estudo permitem também observar que em todas as regiões produtoras e épocas de poda o número de pulverizações necessárias, de acordo com as condições meteorológicas, foi menor do que o número de pulverizações recomendadas pelo sistema do calendário, com a relação entre o $N P_{\text {chuva }}$ e a $N P_{\text {calendário }}$ sendo sempre menor do que $68,42 \%$. Isso demonstra que a racionalização do controle das doenças fúngicas é uma necessidade. A aplicação dos fungicidas nos momentos corretos torna o controle da doença mais eficaz, já que esse controle nas enfermidades da videira é feito basicamente por meio da aplicação de fungicidas sintéticos. Consequentemente, o uso excessivo desses fungicidas, frequentemente, causam problemas relacionados à resistência do patógeno, fitotoxidez e poluição ambiental (PERUCH et al. 2007), os quais são indesejáveis para uma agricultura sustentável.

Sendo assim, o uso de ferramentas para o planejamento do controle de doenças, como o risco climático, e para as tomadas de decisão quanto ao momento

Tabela 6 - Valores de risco climático para a ocorrência de doenças fúngicas na videira Vitis labrusca (RC, \%), em diferentes percentis, nas localidades de Manga (MG), Jales e Jundiaí (SP), para as épocas de poda simuladas

\begin{tabular}{|c|c|c|c|c|c|c|}
\hline \multirow{2}{*}{ Local/Estado } & \multirow{2}{*}{ Poda } & \multicolumn{5}{|c|}{ Percentis } \\
\hline & & $50 \%$ & $70 \%$ & $80 \%$ & $90 \%$ & $95 \%$ \\
\hline \multirow{8}{*}{ Manga, MG } & $01 / \mathrm{Fev}$ & 21,43 & 28,57 & 28,57 & 35,71 & 35,71 \\
\hline & $15 / \mathrm{Fev}$ & 21,43 & 21,43 & 28,57 & 28,57 & 28,57 \\
\hline & 01/Mar & 14,29 & 21,43 & 21,43 & 21,43 & 21,43 \\
\hline & 15/Mar & 7,14 & 14,29 & 14,29 & 14,29 & 21,43 \\
\hline & 01/Abr & 0,00 & 7,14 & 7,14 & 7,14 & 14,29 \\
\hline & 15/Abr & 0,00 & 0,00 & 0,00 & 7,14 & 7,14 \\
\hline & 01/Mai & 0,00 & 0,00 & 0,00 & 7,14 & 7,14 \\
\hline & 15/Mai & 0,00 & 0,00 & 0,00 & 7,14 & 7,14 \\
\hline \multirow{10}{*}{ Jales, SP } & $01 / \mathrm{Fev}$ & 40,00 & 46,67 & 46,67 & 53,33 & 53,33 \\
\hline & $15 / \mathrm{Fev}$ & 40,00 & 40,00 & 46,67 & 46,67 & 53,33 \\
\hline & 01/Mar & 33,33 & 33,33 & 40,00 & 40,00 & 40,00 \\
\hline & 15/Mar & 26,67 & 33,33 & 33,33 & 33,33 & 33,33 \\
\hline & 01/Abr & 20,00 & 26,67 & 26,67 & 33,33 & 33,33 \\
\hline & $15 / \mathrm{Abr}$ & 20,00 & 20,00 & 26,67 & 26,67 & 26,67 \\
\hline & 01/Mai & 13,33 & 20,00 & 26,67 & 26,67 & 26,67 \\
\hline & 15/Mai & 13,33 & 20,00 & 26,67 & 33,33 & 33,33 \\
\hline & 01/Jun & 20,00 & 26,67 & 26,67 & 26,67 & 26,67 \\
\hline & $15 /$ Jun & 20,00 & 26,67 & 33,33 & 33,33 & 33,33 \\
\hline \multirow{6}{*}{ Jundiaí, SP } & $01 / \mathrm{Jul}$ & 36,84 & 36,84 & 42,11 & 47,37 & 47,37 \\
\hline & $15 / \mathrm{Jul}$ & 36,84 & 42,11 & 47,37 & 47,37 & 52,63 \\
\hline & 01/Ago & 42,11 & 47,37 & 47,37 & 57,89 & 57,89 \\
\hline & 15/Ago & 47,37 & 52,63 & 52,63 & 57,89 & 57,89 \\
\hline & $01 /$ Set & 52,63 & 57,89 & 63,16 & 63,16 & 63,16 \\
\hline & $15 /$ Set & 57,89 & 63,16 & 63,16 & 68,42 & 68,42 \\
\hline
\end{tabular}


Tabela 7 - Valores de risco climático para a ocorrência de doenças fúngicas na videira Vitis labrusca (RC, \%), em diferentes percentis, nas localidades de São Miguel Arcanjo (SP), Marialva (PR) e Caxias do Sul (RS), para as épocas de poda simuladas

\begin{tabular}{clccccc}
\hline \multirow{2}{*}{ Local } & \multicolumn{1}{c}{ Poda } & $50 \%$ & $70 \%$ & $80 \%$ & $90 \%$ & $95 \%$ \\
\hline \multirow{4}{*}{ São Miguel Arcanjo, SP } & 01/Jul & 40,00 & 45,00 & 45,00 & 45,00 & 45,00 \\
& 15/Jul & 45,00 & 45,00 & 50,00 & 50,00 & 50,00 \\
& 01/Ago & 45,00 & 50,00 & 50,00 & 55,00 & 55,00 \\
& 15/Ago & 50,00 & 55,00 & 55,00 & 60,00 & 60,00 \\
& 01/Set & 50,00 & 55,00 & 55,00 & 60,00 & 60,00 \\
& 15/Set & 55,00 & 60,00 & 60,00 & 60,00 & 60,00 \\
\hline \multirow{4}{*}{ Marialva. PR } & 01/Jul & 43,75 & 43,75 & 50,00 & 50,00 & 50,00 \\
& 15/Jul & 43,75 & 50,00 & 50,00 & 56,25 & 56,25 \\
& 01/Ago & 50,00 & 56,25 & 56,25 & 56,25 & 62,50 \\
& 15/Ago & 50,00 & 56,25 & 56,25 & 62,50 & 62,50 \\
& 01/Set & 56,25 & 62,50 & 62,50 & 62,50 & 68,75 \\
& 15/Set & 56,25 & 62,50 & 62,50 & 68,75 & 68,75 \\
\hline \multirow{4}{*}{ Caxias do Sul, RS } & 01/Ago & 52,63 & 52,63 & 57,89 & 63,16 & 63,16 \\
& 15/Ago & 52,63 & 52,63 & 63,16 & 63,16 & 63,16 \\
& 01/Set & 52,63 & 57,89 & 63,16 & 68,42 & 68,42 \\
& 15/Set & 52,63 & 57,89 & 63,16 & 63,16 & 68,42 \\
\hline
\end{tabular}

correto de aplicação do controle, como os sistemas de alerta fitossanitário, é primordial para que a agricultura em geral e a viticultura mais especificamente atinjam níveis de excelência, de modo a produzir produtos de melhor qualidade e com menores impactos ao ambiente e aos produtores (WOLF; ISARD, 2007). Além disso, o uso dessas informações possibilita a maximização da eficiência dos controles, já que os elementos meteorológicos, como a temperatura, a umidade relativa e a chuva, interferem na ação dos defensivos agrícolas, assim como reportado para o controle do míldio da videira por Czermainski e Sônego (2004).

Com base nos resultados apresentados nas Tabelas 6 e 7 e considerando-se um nível de probabilidade de $70 \%$ e um limite máximo de RC tolerável de 40\% (moderado), as melhores épocas de poda para a videira Vitis labrusca, com menor pressão de doenças fúngicas, são: de $1^{\circ}$ de fevereiro a 15 de maio em Manga; de $1^{\circ}$ de março a 15 de junho em Jales; e em $1^{\circ}$ de julho em Jundiaí. Nas localidades de São Miguel Arcanjo, Marialva e Caxias do Sul, o RC, em todas as épocas de poda e em todas as classes de probabilidade, é sempre maior do que $40 \%$, o que indica que nessas regiões o cultivo da videira é sempre sob condições de risco climático alto e muito alto, o que exige constante atenção para o manejo das doenças fúngicas. Apesar disso, vale salientar que os maiores RCs nessas áreas estão sempre associados às podas de setembro, a partir de quando as chuvas se intensificam (Figura 1).

\section{CONCLUSÃO}

Os resultados apresentados indicaram haver diferenças no risco climático para a ocorrência de doenças fúngicas na videira Vitis labrusca entre as regiões produtoras do Sul e Sudeste do Brasil. A época de poda afeta consideravelmente o risco climático para ocorrência das doenças nas regiões de Manga, Jales e Jundiaí. Por outro lado, nas regiões de São Miguel Arcanjo, Marialva e Caxias do Sul, a época de poda teve pouca influência nesse risco, sendo sempre classificado como alto a muito alto. O número de pulverizações empregadas pelo sistema do calendário foi invariavelmente maior do que o necessário para o controle do míldio, da antracnose e das manchas das folhas, o que demonstra que a racionalização do uso de defensivos para controle de doenças fúngicas na videira Vitis labrusca é uma necessidade e deve ser baseada em sistemas que levem em consideração pelo menos dados pluviométricos.

\section{REFERÊNCIAS}

ALVARES, C. A.; STAPE, J. L.; SENTElHAS, P. C.; GONÇALVES, J. L. M. Modeling monthly mean air temperature for Brazil. Theoretical and Applied Climatology. DOI 10.1007/s00704-012-0796-6. v. 110, n. 4, p.1-23, 2012. 
BARDIN, L.; PEDRO JÚNIOR, M. J.; MORAES, J. F. L. Risco climático de ocorrência de doenças fúngicas na videira 'Niagara Rosada' para a região do Pólo Turístico do Circuito das Frutas do Estado de São Paulo. Bragantia, v. 69, n. 4, p. 1019-1026, 2010.

CAFFI, T. et al. Empirical vs. mechanistic models for primary infections of Plasmopara viticola. EPPO Bulletin, v. 37, n. 2, p. 261-271, 2007.

CZERMAINSKI, A. B.; SÔNEGO, O. R. Influência das condições climáticas sobre a eficácia de fungicidas empregados para o controle do míldio em Vitis vinífera. Ciência Rural, v. 34, n. 1, p. 1-11, 2004.

LOZADA GARCIA, B. I. et al. Filling in missing rainfall data in the Andes region of Venezuela, based on a cluster analysis approach. Revista Brasileira de Agrometeorologia, v. 14, p. 120-126, 2006.

LOZADA GARCIA, B. I. et al. Climatic risk for potato late blight in the Andes region of Venezuela. Scientia Agricola, v. 65 , p. 32-39, 2008. Número Especial.

ORLANDINI, S.; MASSETTI, L.; DALLA MARTA, A. An agrometeorological approach for the simulation of Plasmopara viticola. Computers and Electronics in Agriculture, v. 64, n. 2, p. 149-161, 2008.

PEDRO JÚNIOR. M. J.; SENTELHAS P. C.; POMMER, C. V. Determinação da temperatura-base, graus-dia e índice biometeorológico para a videira 'Niagara Rosada'. Revista Brasileira de Agrometeorologia, v. 2, n. 1, p. 51-56, 1994.

PEDRO JÚNIOR, M. J.; PEZZOPANE, J. R. M.; MARTINS, F. P. Uso da precipitação pluvial para previsão de épocas de pulverização visando controle de doenças fúngicas na videira 'Niagara Rosada'. Revista Brasileira de Agrometeorologia, v. 7, n. 1, p. 107-111, 1999.

PEREIRA, A. R.; ANGELOCCI, L. R.; SENTELHAS, P. C. Agrometeorologia: fundamentos e aplicações práticas. Guaíba: Editora Agropecuária, 2002. 478 p. v. 1.

PERUCH, L. A. M. et al. Biomassa cítrica, extrato de algas, calda bordalesa e fosfitos no controle do míldio da videira, cv. Niágara Branca. Revista de Ciências Agroveterinárias, v. 6, n. 2, p. 143-148, 2007.

POMMER, C. V. UVA: Tecnologia de Produção, Pós-colheita, Mercado. Porto Alegre: Cinco Continentes, 2003. 777 p. v. 1.

ROSSI, V. et al. Elaboration and validation of a dynamic model for primary infections of Plasmopara viticola in North Italy. Rivista Italiana Agrometeorologia, v. 3, p. 7-13, 2005.

SCHIEDECK, G. et al. Fenologia da videira Niágara Rosada cultivada em estufa e a céu aberto. Revista Brasileira de Agrometeorologia, v. 5, n.2, p. 199-206, 1997.

VALE, F. X. R.; JESUS JÚNIOR, W. C.; ZAMBOLIM, L. Natureza das epidemias. In: VALE, F. X .R. et al. Epidemiologia aplicada ao manejo de doenças de plantas. Belo Horizonte:Perfil, 2004. p. 21-46.

WOLF, E. D.; ISARD S. A. Disease cycle approach to plant disease prediction. Annual Review of Phytopathology, v. 45, p. 203-220, 2007. 